\title{
Dengue Fever: Clinical Spectrum, And Management
}

\author{
Murtaza ustafa ${ }^{1}$, EM.Illzam ${ }^{2}$, MS.Jeffree ${ }^{3}$, RK.Muniandy ${ }^{4}$, S.Husain ${ }^{5}$, MZ.Hud ${ }^{6}$, \\ K.Fairrul $^{7}$, AM.Sharifa ${ }^{8}$.. \\ 1,3,4,5,6,7, Faculty Of Medicine And Health Sciences, University Malaysia Sabah,Kotakinabalu,Sabah,Malaysia \\ ${ }^{2}$ Clinic Family Planning Association, Kota Kinabalu, Sabah, Malaysia \\ ${ }^{8}$ Quality Control Unit, Hospital Queen Elizabeth,KotaKinabalu,Sabah, Malaysia
}

\begin{abstract}
Dengue fever a vector borne disease also transmitted via infected blood products and organ donation. Mainly prevalent in Southeast Asia, now has spread to other countries. Worldwide cost of dengue is estimated US 9 billion. There are five strains (serotypes) of the virus, distinction between serotypes are based on the antigenicity. The course of disease is divided into three phases,febrile, critical, and recovery. The critical phase may proceed to organ dysfunction,severe bleeding and dengue shock-syndrome, and dengue hemorrhagic fever. Diagnosis is made clinically on the reported symptoms, physical examination, with low white blood cell count, low platelet and metabolic acidosis. Treatment depends on symptoms, and maintaining fluid balance is important. Blood transfusion is initiated early in individuals with unstable vital signs. NSAIDS such as ibuprofen and aspirins are avoided, as they might aggravate the risk of bleeding. Prevention by controlling A.aegypti is by eliminating its habitats. Dengue vaccine (Dengavaxia) is available in many countries. There is an urgent need to develop antiviral drugs to treat dengue fever and prevent severe complications.
\end{abstract}

Keywords:Dengue fever, Dengue shock syndrome,Management, Dengue vaccine

\section{Introduction}

Dengue fever is a mosquito borne tropical disease caused by dengue virus-Aedesaegypti[1].The virus has five different types infection with one type usually give lifelong immunity to that type, but only short immunity to others[2].Subsequent infection with a different type increases the risk of severe complications...[1]].Dengue has become a global problem since the Second World War and is common in more than 110 countries[3].Each year between 50 and 528 million people are infected and approximately 10,000 to 20,000 die[4].The earliest description of an outbreak date from 1779[5].Its viral cause and spread was understood by the early $20^{\text {th }}$ century [6,wp,12]Symptoms typically begin three to fourteen days after infection[7].This may include a high fever,headache, vomiting, muscle and joint pains, and characteristic skin rash.[1,7].Recovery generally takes two to seven days[1]In a small proportion of cases, the disease develops into the life threatening dengue hemorrhagic fever, resulting in bleeding, low levels of platelets and blood plasma leakage, or dengue shock syndrome, where dangerously low blood pressure occurs[7].Treatment of acute dengue is supportive and includes giving fluid either oral or intravenously for mild or moderate disease. For severe cases blood transfusion may be required [7].Prevention by reducing mosquito habitat and limiting exposure to bites[1].A vaccine for dengue fever is available in a number of countries[8].Apart from eliminating the mosquitoes, work is ongoing for medication targeted directly at the virus[9].The paper reviews the epidemiology, clinical spectrum, and management of dengue fever.

\section{Historical perspectives}

The first record of a case of probable dengue fever is in Chinese medical encyclopedia from Jin Dynasty(245-420AD) which referred to "water poison" associated with flying insects [5].The primary vector, $A$ aegypti, spread from Africa in the $15^{\text {th }}$ to $19^{\text {th }}$ centuries due in part to increased globalization secondary to the slave trade[10].There have been descriptions of epidemics in the $17^{\text {th }}$ century, but the most plausible early reports of dengue epidemics are from 1779 and 1780,when an epidemic swept across Asia, Africa and North America[5].From that time until 1940,epidemics were infrequent[5].

In 1906, transmission by the Aedes mosquitoes was confirmed, and in 1907 dengue was the second disease (after yellow fever) that was shown to be caused by a virus[6].Further investigations by John Burton Cleland and Joseph Franklin Siler completed the basic understanding of dengue transmission[6].

The marked spread of dengue during after Second World War has been attributed to ecologic disruption. The same trends also led to the spread of different serotypes of the disease to new areas, and to the emergence of dengue hemorrhagic fever.This severe form of the disease to new areas, and to the emergence of dengue hemorrhagicfever. This severe form of the disease was first reported in the Philippines in 1953,by 1970s,it had become a major cause of child mortality and emerged in the Pacific and the Americas[5].Dengue hemorrhagic fever and dengue shock syndrome were first noted in Central and South America in 1981,as 
DENV-2 was contracted by the people who had previously been infected with DENV-1 several years earlier[11].

The origins of the Spanish word dengue are not certain, but it is possibly derived from dinga in the Swahili phrase Ka-dingapepo, which describes the disease as being caused by an evil spirit[12].Slaves in the West Indies having contracted dengue were said to have the posture and gait of a dandy, and disease was known as "dandy fever"[13].The term" break bone-fever"was applied by physician and United States Founding Father Benjamin Rush in 1789 report of 1780 epidemic in Philadelphia. In the report title uses the more formal term: bilious remitting fever"[14].The term dengue fever came into general use only after1882[13].Other historical terms include "break heart fever "and "la dengue"[13].The terms for severe disease include "infectious thrombocytopenic purpura" and "Philippine:, "Thai",or Singapore” hemorrhagic fever[13].

\section{Prevalence}

Most people with dengue recover without any ongoing problems[15].The fatality rate is $1-5 \%[3]$, and less than $! \%$ with adequate treatment[15],however, those who develop low blood pressure may have fatality rate of up to 26\%[3].Dengue is common in more than 110 countries[3].In 2013 it causes about 60 million symptomatic infections worldwide, with $18 \%$ admitted to hospital and about 13,600 deaths[16].The worldwide cost of dengue is estimated US 9 billion[16].For the decade of 2000s, 12 countries in Southeast Asia were estimated to have about 3 million infections and 6,000 deaths annually[17].It is reported in at least 22 countries in Africa; but is likely present in all of them with $20 \%$ of the population at risk[18].This makes it one of the most common vector diseases worldwide [19].

Infections are most commonly acquired in the urban environment\{20].In recent decades, the expansion of villages, towns and cities in the areas in which it is common, and the increased mobility of people has increased the number of epidemics and circulating viruses. Dengue fever, which was once confined to Southeast Asia, has now spread to South China, countries in the Pacific Ocean and America[20], and might pose threat to Europe [21].

Rates of dengue increased 30 fold between 1960-2010[22]. This increase is believed to be due to a combination of urbanization, population growth, increased international travel, and global warming [4].The geographical distribution is around the equator.0f the 2.5 billion people living in areas where it is common $70 \%$ are from Asia and the Pacific[22].An infection with dengue is second only to malaria as a diagnosed cause of fever among travelers returning from the developing world[23].It is the most common viral disease transmitted by arthropods[24], and has a disease burden estimated at 1.600 disability-adjusted life years per million [25].The World Health Oganization counts dengue as one of seventeen neglected tropical diseases[26].

\section{Virus etiology}

Pathogens: Dengue fever (DENV) is an RNA virus of the family Flaviviridae;genusFlavivirus.Othermembers of the same genus include yellow fever virus, West Nile virus, St.Louis encephalitis virus, Japanese encephalitis virus, tick-borne encephalitis virus,Kyasanur forest virus, and Omsk hemorrhagic fever virus[11].Most are transmitted by arthropods(mosquitoes or ticks), and are therefore also referred to as arboviruses(arthropod-borne viruses[11].Dengue virus genome(genetic material) contains about 11,000 nucleotidebases, which code for the three different types protein molecules(C,prM and $\mathrm{E})$ that form virus particle and seven other types of protein molecules (NS1,NS2a,NS2b,NS3,NS4b,NS5) that are found in infected host cells only and are required for replication of the virus[24].There are five[2], strains of the virus called serotypes, of which the first four are referred to as DENV-1,DENV-2,DENV-3,DENV-4[27].The fifth type was announced in 2013 [2].The distinction between the serotypes are based on the antigenicity28].

Transmission: Dengue virus is primarily transmitted by Aedesmosquitoes, particularlyA.aegypti[27].These mosquitoes usually live between the latitudes of $35^{\circ}$ North and $35^{\circ}$ South below an elevation of 1,000 meters(3,300 ft)[27].The typically bite during the early morning and in the evening[29].but they may bite and spread infection at any time of the day[30].Other Aedes species that transmit the disease include A.albopicticus,A.polynesiensis, and A.scutellaris[27].Humans are the primary host the virus[28],but it also circulates in nonhuman primates[31].An infection can be acquired by a single bite[32].A female mosquito that takes a blood meal from a person infected with dengue fever, during the initial 2-10 day febrile period,becomes itself infected with the virus in the cell lining its gut[33].About 8-10 days later, the virus spreads to other tissues including the mosquito's salivary glands and subsequently released into saliva. The virus seems to have no detrimental effect on the mosquito, which remain infected for life[20].Aedesaegypti is particularly involved, as it prefers to lay eggs in artificial water containers, to live in close proximity to humans, and to feed on people rather than other vertebrates[20].

Dengue can also be transmitted via infected blood products and through organ donation [34].In countries such as Singapore, where dengue is endemic, the risk is estimated to be between 1.6 to 6 per 100,000 transfusions[35].Vertical transmission(from mother to child) during pregnancy or at birth has been 
reported[36].Other person-to-person modes of transmission have also been reported, but are very unusual[23].The genetic variation in dengue viruses is region specific, suggestive that establishment into new territories is relatively infrequent, despite dengue emerging in new regions in recent decades[10].

Contributory factors: Severe disease is more common in babies and young children, and in contrast to many other infections, it is more common in children who are relatively well nourished [3,].Other disease factors for severe disease include female sex , high body mass index[10], and viral load[37]. While each serotype can cause the full spectrum of disease[24], virus strain is a risk factor[10].Infection with one serotype is thought to produce lifelong immunity to that type, but only short-term protection against other three[28,23].The risk of severe disease from secondary infection increases if someone previously exposed to serotypes DENV-1,DENV2[25].Dengue can be life threatening in people with chronic diseases such as diabetes and asthma[25].Polymorphism(normal variation) in particular genes have been linked with an increased risk of severe dengue complications. Examples include the genes coding for proteins known as TNF $\alpha$,mannanbindind lectin[4],CTLA4,TGF $\beta$ [28,rept],DC-SIGN,PLCE1, and particular forms of human leukocyte antigen from gene variations of HL-A-B[10,25].A common genetic abnormality, especially in Africans, known as glucose-6phosphate dehydrogenasedeficiency,appears to increase the risk [37].Polymorphisms in the genes for vitamin D receptor and FcyR to offer protection against severe disease in secondary dengue infection [25].

Infection process: When a mosquito carrying dengue virus bites a person, the virus enters the skin together with the mosquito's saliva. It binds and enters white blood cells, and reproduces inside the cells while the move throughout the body. The white blood cells respond by producing a number of signaling proteins, such as cytokines and interferons, which are responsible for many of the symptoms, such as the fever, the flu -like symptoms, and the severe pains. In severe infection, the virus production inside the body is greatly increased, and many more organs (such as the liver and bone marrow) can be affected. Fluid from the bloodstream leaks through the wall of small blood vessels into body cavities due to capillary permeability. As a result, less blood circulated in the blood vessels, and blood pressure becomes low that it cannot supply sufficient blood to vital organs. Furthermore, dysfunction of the bone marrow due to infection of the stromal cells leads to reduced number of platelets, which are necessary for effective blood clotting, this increases the risk of bleeding, the other major complication of dengue fever[37].

Viral replication: Once the inside the skin, dengue virus binds to Langerhans cells (a population of cells in the skin that identifies the pathogens)[37].The virus enters the cells through binding between viral proteins and membrane protein on the Langerhans cell, specifically the C-type lectins called DC-SIGN, mannose receptor and CLES5A[24t].DC-SIGN-a non-specific receptor for foreign material on dendritic cells, seems to be the main point of entry[25]. The initial reaction of infected cells is to produce interferon, a cytokine that raises a number of defenses against viral infection through innate immune system by augmenting the production of a large group of proteins mediated by the JAK-STAT pathway[24].Various antibodies are generated, some bind closely to the viral proteins and target them fir phagocytosis, but some bind the virus less well and appear instead to deliver the virus into a part of the phagocytes where it is not destroyed but is able to replicate further[24].

Dengue shock syndrome (DSS): It is not clear why secondary infection with a different strain of dengue virus places people at risk of dengue hemorrhagic shock syndrome. The most widely accepted hypothesis is that of antibody-dependent enhancement (ADE). The exact mechanism behind ADE is unclear. It may be caused by poor binding of non-neutralizing antibodies and delivery into the wrong compartment of white blood cells that have ingested the virus for destruction[24,25].There is suspicion that ADE is not the only mechanism underlying severe dengue-related complications[4,38], and various lines of research have implied a role for $\mathrm{T}$ cells and soluble factor such as cytokines and the complement system[37].

Severe disease is marked by the problems of capillary permeability((an allowance of fluid and protein normally contained within blood to pass) and disordered blood clotting[39,17,10]. These changes appear associated with a disordered state of the endothelial glycocalyx, which acts as a molecular filter of blood components[10,].Leaky capillaries(and the critical phase) are thought to be caused by an immune system response[10].Other processes of interest include infected cells that becomes necrotic-which affect both coagulation and fibrinolysis(the opposing system of blood clotting and clot degradation)-and low platelets in the blood, also a factor in normal blood clotting (37].

\section{Clinical spectrum}

Typically, people infected with dengue virus are asymptomatic'(80\%) or have only mild symptoms such as an uncomplicated fever[4,27,21]. Other have more severe illness( 5\%), in a small proportions it is lifethreatening[4,21]..The incubation period ranges from 3 to 14 days, but most often it is 4 to 7 days[20].Therefore travelers returning from endemic areas are unlikely to have dengue fever or other symptoms start more than 14 days after arriving home[3].Children often experience symptoms similar to those of the common cold and 
gastroenteritis(vomiting and diarrhea)[39], and have a greater risk of severe complications[3,10],though initial symptoms are generally mild but include high fever[10].

Disease process: The characteristic symptoms of dengue are sudden-onset fever, headache (typically located behind the eyes), muscle and joint pains, and a rash. The alternative name for dengue,"breakbone fever" comes from the associated muscle and joint pains[4,23]. The course of disease is divided into three phases, febrile, critical, and recovery[40]. The febrile phase involves high fever, potentially over $40{ }^{0} \mathrm{C}\left(104^{\circ} \mathrm{F}\right)$, and is associated with generalized pain and a headache; this usually lasts two to seven days[41,23].Nausea and vomiting may also occur[10t].A rash occurs in 50-80\% of those with symptoms[23,41],in the first or second day of symptoms as flushed skin. Or later in the course of illness(days 4-7), as measles-like rash[41,,42].A rash described as :island of white in sea of red" has also been described[11].Some petechiae (small red spots that do not disappear when skin is pressed, which are caused by broken capillaries) can appear at this point[41], as some mild bleeding from mucous membrane of the mouth and nose[3,23].The fever itself is classically biphasic or saddleback in nature, breaking then returning for one or two days[42,11].

Critical phase: In some individuals the disease may proceeds to a critical phase as fever resolves [10].During this period, there is leakage of plasma from blood vessels, typically lasting one to two days[40].This may result in fluid accumulation in the chest and abdominal cavity as well as depletion of fluid from the circulation and decreased blood supply to vital organs[40]. There may also be organ dysfunction and severe bleeding, typically from gastrointestinal tract[3,40].Shock(dengue shock-syndrome)and hemorrhage(dengue hemorrhagic fever) occur in less than 5\% of all cases [3], however, those who have previously infected with other serotypes of dengue fever(secondary infection) are at increased risk[3,24].This critical phase, while rare occurs relatively more commonly in children and young adults[10].

Recovery phase: The recovery phase occurs next, with resorption of leaked fluid into the bloodstream[41].This lasts two to three days[3].The improvement is often striking, and can be accompanied with severe itching and slow heat rate[3,40]. Another rash may occur with either maculopapular or vasculitis appearance, which is followed by peeling of skin[[10].During this stage, a fluid overload state may occur; it effects the brain, it may cause a reduced level of consciousness or seizures[3].A feeling of fatigue may last for weeks in adults[10].

Related problems: Dengue can occasionally affect severely other body systems [40], either in isolation or along with other classic dengue symptoms[39].A decreased level of consciousness occurs in $0.5-6 \%$ of severe cases, which is attributable either to inflammation of the brain by the virus or indirectly as a result of impairment of vital organs, for example, the liver[40,11,38].Other neurological disorders have been reported in the context of dengue, such as transverse myelitis and Gullian-Barre syndrome [39,38].Infection of heart and acute liver failure are among the rarer complications[3,40].A pregnant woman who develops dengue fever may be at higher risk of miscarriage as well as low birth weight and premature birth[43].

\section{Diagnosis}

The diagnosis of dengue is typically made clinically, on the basis of reported symptoms and physical examination; this applies especially in endemic areas[4].A probable diagnosis is based on the findings of fever plus two other findings e.g. Nausea and vomiting, rash, generalized pain, low blood cell count, and positive tourniquet test.Vital signs or(warning signs) include worsening abdominal pain, ongoing vomiting, liverenlargement, mucosal bleeding, high hematocrit with low platelets, lethargy and restlessness, and serosal effusion[10,15].

Vital signs typically occur before the onset of severe dengue [40]. The tourniquet test, which is particularly useful in settings where no laboratory investigations are readily available[40]. The diagnosis should be considered in anyone who develops a fever within two weeks of being in the tropics[10].It can be difficult to distinguish dengue fever and chikungunya, a similar viral infection that shares many symptoms and occurs in similar parts of the world to dengue[23].Often, investigations are performed to exclude other conditions that cause similar symptoms, such as malaria, leptospirosis, viral hemorrhagic fever, typhoid fever, meningococcal disease, measles, and influenza[3,44].Zika fever also has similar symptoms as dengue [45].

The earliest change detectable on laboratory investigations is low white blood cell count, which may then be followed by low platelet and metabolic acidosis [3].A moderately elevated level of aminotransferase(AST and ALT) from liver is commonly associated with low platelet and white blood cells[[10].In severe disease, plasma leakage results in hemo-concentration(as indicated by a rising hematocrit)and hypoalbuminemia[3].Dengue shock syndrome is present if pulse pressure drops to $\leq 20 \mathrm{~mm} \mathrm{Hg}$ along with peripheral vascular collapse[10].Dengue fever was graded into grades 1-IV.Grades 111 and IV are referred to as "dengue shock syndrome"[15].

The diagnosis of dengue fever may be confirmed by microbiological laboratory testing[15,46].This can be done by virus isolation in cell cultures, nucleic acid detection by PCR, viral antigen detection such as for NSI or specific antibodies (serology) [25,44].All tests may be negative in the early stages of the disease[3,25,].PCR 
and viral antigen detection are more accurate in first seven days[10].The laboratory tests are only diagnostic value during the acute phase of the illness with the exception of serology[20,23,25].

\section{VII.Management}

There are no specific antiviral drugs for dengue, however maintaining proper fluid balance is important [10].Treatment depends on the symptoms[47].Intravenous hydration, if required, is typically only needed for one or two days[47].In children with shock due to dengue a rapid dose of $20 \mathrm{ml} / \mathrm{kg}$ is reasonable[48,60].Invasive medical procedures such as nasogastric intubation, intramuscular injections and arterial procedures are avoided in view of the bleeding risk[3].Paracetamol (acetaminophen) is used for fever and discomfort while NSAIDs such as ibuprofen and aspirins are avoided as they might aggravate the risk of bleeding[47].Blood transfusion is initiated early in people presenting with unstable vital signs in the face of decreasing hematocrit, rather than waiting for hemoglobin concentration to decrease to some predetermined "transfusion trigger" level[3].Packed red blood cells or whole blood are recommended, while platelets and fresh frozen plasma are usually not[49].There is not enough evidence to determine if corticosteroids have a positive or negative effect in dengue fever[50].During the recovery phase intravenous fluids are discontinued to prevent a state of fluid overload[3].If fluid overload occurs and vital signs are stable, stopping further fluid may be all that is needed[49].If a person is outside of the critical phase, a loop diuretic such as furosemide may be used to eliminate excess fluid from the circulation[47].

\section{Prevention}

Prevention depend on control of and protection from bites of the mosquitos that transmit it[29,51].The World Health Organization recommends and Integrated Vector Control program consisting of five elements[29],a) Advocacy, social mobilization and legalization to ensure that public health bodies and communities are strengthened, b)Collaboration between the health and other sectors(public and private ,c) An integrated approach to disease control to maximize use of resources.d)Evidence-based decision making to ensure any interventions are targeted appropriately, and e)Capacity -building to ensure an adequate response to the local institution.

The primary method of controlling A aegypti is by eliminating it habitat[29].This done by getting rid of open sources of water, or it is not possible, by adding insecticides or biological control agents to these areas[29].Generalized spraying with organophosphate or parathyroid insecticides, while sometimes done, is not thought to be effective[21].People can prevent mosquito bites by wearing clothing that fully covers the skin,using mosquito netting and application of insect repellent(DEET) being most effective[32].In 2016 a partially effective vaccine for dengue fever became available in the Philippines and Indonesia[8,52].The vaccine is produced by Sanofi and goes by the trade name Dengavaxia[53].It is based on a weakened combination of yellow fever virus and each of four dengue serotypes[52]. Vaccine 60\% effective and prevented more than 80 to $90 \%$ of severe cases[54].One of the concerns is that a vaccine could increase the risk of severe disease through antibody-dependent enhancement(ADE)[55].Ideal vaccine is safe, effective after one or two injections, covers all serotypes, does not contribute ADE ,is easily transported and stored and is both affordable and cost effective[55].

\section{Future directions}

Research efforts to prevent and treat dengue include various means of vector control [56],vaccine development, and antiviral drugs[51].Some novel methods are used to reduce mosquito numbers by placement of the guppy(Poecilia reticulate) or copepods in standing water to eat mosquito larvae[56].Ferlien and colleagues concluded that ethanolic leaf extract of rambutan (Nepheliumlappaceum) can be used as a mean of terminating the growth and development of the larvae of house hold mosquito [57].There are also trials with genetically modified male A.aegypti that after release into the wild mate with females, and render their offspring unable to fly[58].Attempts are ongoing to infect the mosquito population with bacteria of the Wolbachiagenus, which makes the mosquitoes resistant to dengue virus[10,59]There are ongoing efforts to develop antiviral drugs that would be used to treat attacks of dengue fever and prevent severe complications[9,60].Discovery of the structure of the viral protein may aid the development of effective drugs[9].Finally, it may be possible to develop entry inhibitors, which stop the virus entering cells or inhibitors of 5' capping process, which is required for viral replication[60].

\section{Conclusions}

Dengue fever occurs mainly in the tropics and among travelers returning from the endemic regions. Treatment of acute form is supportive with fluid either oral or intravenously, and blood transfusion for severe cases. Prevention by vector control, exposure to bites, 
and adding insecticides or biological control of open spaces of water. Effective dengue vaccine is available in many countries.

\section{References}

[1]. Dengue and severe dengue Fact Sheet. $N^{0} 117$.WHO May 2015.Retrived 3 February 2016.

[2]. NormileD.Surprising new dengue virus throws a spanner in disease control efforts.Science.2013.342 (6157):415.

[3]. RanjitS,KissonN.Denguehemorrahagic fever and shock syndrome. PediatrCrit Care Med.2011;12)1)1:90-100.

[4]. WhitebornJ,Farrar J.Dengue.Br Med Bull.2010;95:161-73.

[5]. GublerDJ.Dengue and dengue hemorrhagic fever.ClinMicrobiol Rev.1998;11(3):480-96.

[6]. HenchalEA,PutnakJR.The dengue viruses..ClinMicrobiol Rev.1990;3(4):376-96.

[7]. KularatneSA.Denguefever.BMJ(Clinical research ed.)2015;351:h4661.

[8]. East Susie(6 April 2016).World's first dengue fever vaccine launched in the Philippines.CNN.Retrieved 17 October 2016.

[9]. Noble CG.ChenYL,DongH, etal.Strategies for development of Dengue virus inhibitors. Antiviral Res.2010;85(3):450-62.

[10]. Simmons CP,FarrarJJ,NguyenvV,etal.Dengue.NEngl J Med.2012;366(15):1423-32.

[11]. Gould EA,SolomonT.Pathogenicflaviviruses.The Lancet.2008;371(9611):500-9.

[12]. Anonymour(15,June 1998).Definition of Dandy fever.MedicineNet.com.Retrieved 25 December 2010.

[13]. Halstead SB(2008),Dengue(Tropical Medicine:Science and Practice).River Edge.N J:Imperial College Press.PP.1-10.ISBN 184816-228-6.

[14]. Barrett AD.StansburyLR(2009).Vaccine for biodefense and emerging and neglected diseases.San Diego:Academic.pp.287323.ISBN 0-12-369408-6.

[15]. WHO(2009),pp.10-11.

[16]. Shepard DS,UndurragaEA,HalasaYA,etal.The global economic burden of dengue: a systematic analysis.TheLancet.InfectiousDiseases.2016;16(8):935-41.

[17]. Shepard DS,UndurragaEA,HalasaYA,etal.Economic and disease burden of dengue in Southeast Asia.PLoSNegl TropDis.2013;7(2):e2055.

[18]. AmarasingheA,KritskJN,LestonGW,etal.Dengue virus infection in Africa.Emerging Infectious Diseases.2011;17(8):1349-54

[19]. YacoubSohie,WillisBridget.Predicting outcome from dengue.BMC Medicine. 2014; 12(1):147.

[20]. GublerDJ(2010).Dengue viruses.InMahyBWJ;VanRegenmortelMHV.Desk Encyclopedia of Human and Medical Virolog.Boston::Academic Press.pp.372-82.ISBN 0-12-375147-0.

[21]. Reiter P..Yellow fever and dengue:a threat to Europe.EuroSurveill. 2010; 15(10): 19509 PMID 20403310.

[22]. $\quad \mathrm{WHO}(2009), \mathrm{p} .3$.

[23]. Chen LH,WilsonME.Dengueandchikugunya infections in travelers.Current Opinion in Infectious Diseases.2010;23(5):438-44.

[24]. Rodenhuis-ZybertLA,WilschutJ,AmitJM.Dengue virus life cycle:viral and host factors modulating activity.CellMolLife.2010;67(16):2773-86.

[25]. Guzman MG,HalsteadSB,ArtsobH,etal.Dengue: a continuing global threat.Nature Reviews Microbiology.2010;8(12 Suppl):S7-S16

[26]. Neglected Tropical Diseases. The 17 neglected tropical disease.World Health Organization. Retrieved 10 April 2013.

[27]. WHO(2009).pp.14-16

[28]. SolomonidesTony(2010).Healthgrid applications and core technologies:procceding of HealthGrid 2010(OnlineAug.ed.)Amsterdam.IOS Press.P.235.ISBN 978-!-60750-582-2.

[29]. WHO(2009).pp.59-64.

[30]. Travellers Health Break Notice.Centers for Disease Control and Prevention.2 June 2010.Archieved from original on 26 August 2010.Retrived 27 August 2010.

[31]. Vector- borne viral infections. World Health Organization.Retrieved 17 January 2011.

[32]. Center for Disease Control and Prevention.Chapter 5-dengue fever(DF) and dengue hemorrhagic fever(DHF).2010.Yellow Book.Retrieved 23 December 2010.

[33]. St.GeorgievVassil(2009).National Institute of Allergy and Infectious Diseases.NIH(1 ed.)Totowa,N.J.Humana.p.268.ISBN 978-160327-297-1.

[34]. Wilder Smith A.,ChenLH.MassadE,etal.Threat of dengue to blood safety in dengue-endemic countries.Emerg InfectDis.2009;16(1):8-11.

[35]. TeoD,NgLC,Lam S.Is dengue a threat to blood supply.Trnsfus Med.2009;19(2):66-77.

[36]. Wiwanitkit V. Unusual mode of transmission of dengue.J Infect Develop Count. 2010;4(1):51-4.

[37]. Martina BE,KorkaP,OsterhausAD.Dengue virus pathogenesis: an integrated view. ClinMicrobiol Rev.2009;22(4):564-81.

[38]. Carod-ArtalFJ,Wichmann 0,Farrar J,etal.Neurological complications of dengue virus infection.Lancet Neurol.2013;12(9):906-19.

[39]. Varatharaj A. Encephalitis in the clinical spectrum of dengue infection. Neurol. India. 2010;58(4):585-91.

[40]. WHO(2009).pp.25-27.

[41]. Wolff K, Johnson RA,eds.(2009).Viral infections of skin and mucosa.Fitzpatrik's color atlas and synopsis of clinical dermatology $\left(6^{\text {th }}\right.$ ed.)New York-Hill Medical.pp.810.2.

[42]. KnoopKJ,StackLB,StorrowA, et al.(2010).Tropical medicine.Atlas of emergency medicine(3 ${ }^{\text {rd }}$ ed.)New York:McGraw-Hill Professional.pp.658-9.ISBN 0-07-149618-1.

[43]. PaixaoES,TeixeiraMG,CostaMD,etal.Dengue during pregnancy and adverse fetal outcomes:a systematic review and metaanalysia.The Lancet Infectious Diseases.2016;16(7):857-865.

[44]. WHO(2009).pp.90-95.

[45]. MussoD,NillesEJ,Cao-Lormeau,etal.Rapid spread of Zika virus in the Pacific area.ClinMicrobiol Infection.2014;20(10):0595-96.

[46]. WiwanitkitV.Dengue fever diagnosis and treatment.Expert review of anti-infective therapy.2010;8(7):841-5.

[47]. WHO (2009),pp.32-37.

[48]. de Caen AR,BergMD,Chameides L, et al.Part 12:Pediatric Advanced Life Support:2015 American Heart Association GuidelinesUpdate for CardiopulmonaryResuscitation andEmergency Cardiovascular Care.Circulation.2015;132(18 Suppl 2):S526-42.

[49]. WHO (2009),pp.40-43.

[50]. Zhang F,KramerCV.Croticosteroids for dengue infection. Dengue fever vaccine Available in Indonesia. October 17,2016.The Cochrane database of systematic reviews.2014;7(7):CD003488

[51]. WHO (2009),p.137-46.

[52]. Dengue fever vaccine available in Indonesia.October 17,2016.

[53]. Dengavaxia ${ }^{\circledR}$, World First Dengue Vaccine,Approved in Mexico. www.sanofipasture.com.Retrieved 2015-12-10.

\begin{tabular}{llr}
\hline DOI: $10.9790 / 0853-1602015359$ & www.iosrjournals.org & $58 \mid$ Page
\end{tabular}


[54]. VillarLuis,Dayan Gustavo Horacio,ArredondoGracia,etal.Efficacy of a Tetravalent Dengue Vaccine in Children in Latin America.NEngl J Med.2014;372(2):113-23.

[55]. Webster DP,FarrarJ,Rowland -Jones S.Progress towards a dengue vaccine.Lancet Infect Dis.2009;9(11):678-87.

[56]. WHO (2009),p.71.

[57]. Ferlien Mae GB,CherrieGM,LourivyPD,etal.Larvicidal Potential of Rambutan(nepheliumlappaceum 1)Leaf extract against household mosquito larvae.http ://research.edu.ph/ojs/index.php/optima/article/view/567.

[58]. Fong I.(2013).Challenges in Infectious Diseases.Springer.p.219.ISBN 978-1-4614-4496-1.

[59]. Bug could combat dengue fever.BBCNEWS.British Broadcasting Corporation.2 January 2009.

[60]. SampathA,PadamanabhanR.Molecular target targets for flavivirus drug discovery. Antiviral Res.2009;81(1):6-15. 